\title{
Propositions méthodologiques pour mesurer la territorialisation des projets de développement rural
}

Anne-Sophie Barbarot, Tiphaine Renard, Dominique Vollet, Denis Lepicier, Marielle Berriet-Solliec et David Tiveyrat

\section{(2) OpenEdition}

1 Journals

Édition électronique

URL : http://journals.openedition.org/economierurale/3985

DOI : 10.4000/economierurale.3985

ISSN : 2105-2581

Éditeur

Société Française d'Économie Rurale (SFER)

Édition imprimée

Date de publication : 15 juillet 2013

Pagination : 81-88

ISSN : 0013-0559

\section{Référence électronique}

Anne-Sophie Barbarot, Tiphaine Renard, Dominique Vollet, Denis Lepicier, Marielle Berriet-Solliec et David Tiveyrat, « Propositions méthodologiques pour mesurer la territorialisation des projets de développement rural », Économie rurale [En ligne], 336 | juillet-août 2013, mis en ligne le 15 juillet 2015 consulté le 04 mai 2019. URL : http://journals.openedition.org/economierurale/3985 ; DOI : 10.4000/ economierurale.3985 


\title{
FAITS ET CHIFFRES
}

\section{Propositions méthodologiques pour mesurer la territorialisation des projets de développement rural}

\author{
Anne-Sophie BARBAROT • Agence de Services et de Paiement, Clermont-Ferrand \\ Tiphaine RENARD • Agence de Services et de Paiement, Clermont-Ferrand \\ Dominique VOLLET • Irstea, UMR Métafort, Clermont-Ferrand \\ Denis LÉPICIER • AgroSup Dijon, CESAER, Dijon \\ En collaboration avec \\ Marielle BERRIET-SOLLIEC et David TIVEYRAT
}

\begin{abstract}
Une méthode de mesure du degré de territorialisation des projets de développement rural est proposée en utilisant les données de la base OSIRIS relatives aux axes 3 et 4 du deuxième pilier de la Politique agricole commune. Une première application aux 21 Groupes d'Action Locale des régions Auvergne et Bourgogne met en évidence, d'une part, une territorialisation plus marquée mais faible du programme Leader et, d'autre part, des différences significatives entre mesures.
\end{abstract}

A u cours de la période 2007-2013, la politique de développement rural de l'Union européenne, relative à sa composante, autrement dit le deuxième pilier de la Politique agricole commune (PAC), est régie par le Règlement de développement rural (RDR) et financée par le Fonds européen pour l'agriculture et le développement rural (FEADER). Cette politique européenne est structurée en quatre axes dont les deux premiers sont davantage orientés vers la régulation sectorielle agricole tandis que les deux derniers (axe 3 et axe 4) sont particulièrement destinés au développement rural. L'axe 4 est considéré comme un axe transversal à dimension méthodologique visant à encourager la mise en œuvre des projets Leader. À l'initiative d'un Groupe d'action locale (GAL), ces projets LEADER correspondent à une programmation, de mesures pour encourager et soutenir des projets infrarégionaux dans le périmètre d'action du GAL. Sur leurs territoires d'action, les GAL peuvent ainsi activer les différentes mesures des trois premiers axes, dans le cadre d'une démarche ascendante et concertée. Toutefois, en complément des projets localisés dans le même périmètre des GAL peuvent être financés par ces mêmes mesures dans le cadre de procédure régionale mise en œuvre de façon plus descendante. Avec une perspective de révision budgétaire de la PAC à la baisse, les acteurs ruraux sont inquiets pour le maintien des soutiens européens au développement rural (Aubert et al., 2011) ${ }^{1}$. De plus, l'intérêt des démarches territoriales portées par l'axe 4 du Feader, coûteuses en animation, est souvent remis en cause (Balny et al., 2010 $)^{2}$. Un grand nombre d'études de cas ponctuelles mettent en évidence ce qui est appelé « la valeur ajoutée » des démarches

1. Aubert F. et Trouvé A. (2011). Politique de développement rural et second pilier de la PAC : sortir de l'ambiguïté pour relever les défis territoriaux de l'agriculture. In J.-P. Butault et al., Face aux nouveaux enjeux : quelles politiques agricoles pour quels systèmes de production? INRA.

2. Balny P., Bobenriether O., Martin De Lagarde O. (2010). Rapport de mise en ouvre des axes 3 et 4 du FEADER, s.1., CGAAER, n 2091. 
territoriales, notamment Leader (Méasson, 2007) ${ }^{3}$. Toutefois, jusqu'à présent, aucune démarche visant à mobiliser des données quantitatives exhaustives n'a été menée. Dans ces conditions, il nous est apparu important de proposer une telle démarche à partir des données concernant l'axe $3 \mathrm{du}$ Feader (voir encadré 1) contenues dans la base OSIRIS (extranet partagé par les services déconcentrés du ministère de l'Agriculture et l'Agence de services et de paiement (ASP) dans lequel sont saisis tous les dossiers du RDR 2) ${ }^{4}$ à partir de l'expérience de deux régions (Auvergne et Bourgogne) engagées dans cette réflexion. Ce travail vise à répondre à la question suivante en se basant sur l'exploitation de la base de données OSIRIS : une politique territoriale telle que Leader présente-t-elle une valeur ajoutée par rapport à des mesures mises en place de façon plus descendante ? Dans ce but, les mêmes mesures de l'axe 3 ont été retenues en comparant des conditions d'application différentes (plutôt « ascendantes » dans le cadre de Leader et plutôt « descendantes » dans un cadre hors Leader). L'objectif est double : d'une part proposer une démarche susceptible d'être appliquée dans d'autres contextes (territoriaux, politiques publiques), d'autre part apporter des éléments de réflexion sur les intérêts des politiques territorialisées.

Pour cela, nous nous sommes appuyés sur le cadre général d'analyse proposé par Trouvé $(2007)^{5}$ et Berriet-Solliec et al.

3. Measson L. (2011). Évaluer l'ingénierie territoriale pour évaluer la plus-value de la territorialisation. L'exemple des politiques agro-environnementales. Colloque SFE «Évaluation des politiques publiques devant les défis d'une société en tension », Nantes.

4. Ces données peuvent être accessibles par les chercheurs via l'Observatoire du développement rural.

5. Trouvé A. (2007). Le rôle des régions européennes dans la redéfinition des politiques agricoles. Université de Bourgogne, Thèse de doctorant, UFR de sciences économiques.
$(2008)^{6}$ sur les facteurs et degré de territorialisation des politiques agricoles, puis sur une grille d'analyse formalisée et testée au niveau de chaque projet par Michel $(2010)^{7}$ et Gloria $(2011)^{8}$.

\section{Une démarche de quantification originale basée sur l'analyse des processus de territorialisation}

Berriet-Solliec et al. (2008) identifient trois principaux processus de territorialisation de l'intervention publique agricole : le renouvellement des procédures de décision impliquant davantage les acteurs locaux ; l'adaptation aux spécificités locales permettant aux politiques publiques territoriales d'être plus efficaces pour prendre en compte les ressources et les demandes spécifiques ; la compensation des effets du marché par exemple pour rémunérer des externalités positives. Ces processus renvoient à quatre leviers principaux (Michel, 2010) : l'ancrage territorial (1) du projet plus ou moins adapté aux spécificités locales ; l'intersectorialité (2) du projet et les coordinations entre acteurs (3) qui constituent toutes deux des formes de gouvernance renouvelées ; le caractère transversal (4) du projet, c'est-à-dire sa capacité à prendre en compte différentes dimensions du développement durable comme la rémunération des externalités positives ou

6. Berriet-Solliec M., Déprés C., Trouvé A. (2008). $\mathrm{La}$ territorialisation de la politique agricole en France : vers un renouvellement de l'intervention publique en agriculture ? In Laurent C., Du Tertre C. (dir.), « Secteurs et territoires dans les régulations émergentes », Paris, L'Harmattan, $\mathrm{n}^{\circ}$ 6, p. 121-136. 7. Michel S. (2010). Territorialisation des politiques de développement rural : analyse comparée des modalités de mise en ouvre des axes 3 et 4 du PDRH (2007-2013) en Auvergne et en Bourgogne. Mémoire de fin d'étude d'ingénieur, AgroSup Dijon, 83 p.

8. Gloria M. (2011). Territorialisation des politiques de développement rural. Analyse comparée de la valeur ajoutée territoriale du programme Leader. Mémoire de fin d'étude d'ingénieur, AgroSup Dijon, 102 p. 


\section{Encadré 1. Rappel des 4 axes du Feader de la programmation 2007-2013}

Axe 1 - Compétitivité de l'agriculture et de la sylviculture (134 millions d'euros, soit $17 \%$ de l'enveloppe en Auvergne ; 85 millions, soit $25 \%$ de l'enveloppe en Bourgogne).

Axe 2 - Environnement et gestion de l'espace (598 millions, soit $77 \%$ de l'enveloppe en Auvergne ; 221 millions, soit $64 \%$ de l'enveloppe en Bourgogne).

Axe 3 - Diversification de l'économie et qualité de vie (25 millions, soit $3 \%$ de l'enveloppe en Auvergne ; 25 millions, soit $7 \%$ de l'enveloppe en Bourgogne).

- Qualité de vie : services pour l'économie, rénovation des villages, appui/formation, appui aux stratégies de développement local.

- Diversification économique : aides aux micro-entreprises, tourisme, conservation du patrimoine naturel et vernaculaire.

Axe 4 - Leader (19 millions, soit $2 \%$ de l'enveloppe en Auvergne ; 14 millions, soit $4 \%$ de l'enveloppe en Bourgogne).

- Démarche participative de développement rural associant les différentes activités du monde rural ; cette démarche concerne les trois axes précédents et est portée par les Groupes d'action Locale (GAL).

la contribution à l'insertion économique et sociale de populations défavorisées.

Les quatre leviers de la territorialisation ont été déclinés en indicateurs pouvant être renseignés à partir des données disponibles dans la base Osiris pour l'ensemble des projets financés par le Feader (tableau 1). Une méthode de calcul a été élaborée afin de pouvoir quantifier la contribution des différents leviers à la valeur ajoutée territoriale des projets et établir des comparaisons. La méthode combine deux niveaux d'analyse, celui du territoire Leader (21 GAL), niveau auquel sont hiérarchisés les enjeux de développement, et celui des projets financés par le Feader, niveau auquel sont quantifiés les critères d'évaluation du degré de territorialisation (Tiveyrat, 2011) .

\section{Quantification de I'ancrage territorial}

Pour chacun des 21 GAL, un diagnostic a été réalisé à l'aide de données Insee relatives à l'emploi, l'activité agricole, etc. (fichiers UNEDIC, Recensement général

9. Tiveyrat D. (2011). Analyse comparée quantitative des degrés de territorialisation des axes 3 et 4 en Auvergne et en Bourgogne. Mémoire de Master 1 Développement des Territoires et Nouvelles Ruralités, Université Blaise Pascal, Clermont-Ferrand. agricole), pour déterminer l'importance des enjeux suivants : maintien et développement des activités agricoles (à partir de l'évolution de la surface agricole utile et du nombre d'exploitations, et de la part du secteur agricole dans l'emploi), des activités industrielles et artisanales (à partir du taux de création d'établissements et des taux de chômage et d'emploi), et enfin des services (à partir de la densité des services marchands, non marchands, et de santé). Une note de 1 à 3 a été attribuée à chaque GAL en regard des valeurs obtenues (faibles, moyennes ou fortes) pour chacune des variables. Cette note (variable selon les territoires) a ensuite été utilisée comme pondération pour estimer l'ancrage territorial d'un projet à partir d'un dépouillement simple (valeur 0 ou 1) de chacun des projets (les items Osiris utilisés étant l'intitulé et le thème principal).

\section{Quantification des autres leviers}

Les critères des leviers intersectorialité, coordination entre acteurs, et transversalité ont été redéfinis en fonction des données pertinentes et quantifiables disponibles à l'échelle des projets : les données ont été extraites de la base de données Osiris et retraitées par l'ASP. Pour ces autres leviers, 
Tableau 1. Critères d'évaluation du degré de territorialisation

\begin{tabular}{|c|c|}
\hline Levier & Critère \\
\hline \multirow{3}{*}{ 1. Ancrage territorial } & $\begin{array}{l}\text { Le projet répond-il à un enjeu de maintien et développement des activités } \\
\text { économiques du territoire? }\end{array}$ \\
\hline & $\begin{array}{l}\text { Le projet répond-il à un enjeu de maintien et développement des services } \\
\text { du territoire? }\end{array}$ \\
\hline & Le projet répond-il à un enjeu de valorisation et protection du patrimoine? \\
\hline \multirow{3}{*}{ 2. Intersectorialité } & Combien de thématiques sont concernées par le projet ? \\
\hline & Combien de cofinanceurs participent au projet ? \\
\hline & $\begin{array}{l}\text { Le projet est-il innovant ou met-il en place une dynamique nouvelle sur le } \\
\text { territoire? }\end{array}$ \\
\hline \multirow{3}{*}{ 3. Coordination entre acteurs } & Le projet vise-t-il à coordonner les différents acteurs du territoire? \\
\hline & Le projet est-il coordonné en interne? \\
\hline & Quel est le potentiel de coordination externe sur le territoire du projet ? \\
\hline 4. Transversalité & $\begin{array}{l}\text { Combien de dimensions du développement durable sont concernées par le } \\
\text { projet? (économique, social, environnemental) }\end{array}$ \\
\hline
\end{tabular}

Source : les auteurs.

Tableau 2. Méthode générale d'estimation du degré de territorialisation (détaillée pour la variable Ancrage territorial) et exemple d'application au GAL du Velay ${ }^{10}$

\begin{tabular}{|c|c|c|c|c|c|c|}
\hline \multicolumn{4}{|c|}{$\begin{array}{l}\text { Méthode générale d'estimation } \\
\mathrm{i}=\text { Territoire } \mathrm{i} \\
\mathrm{n}=\text { Nombre de dossiers sur le territoire }\end{array}$} & \multicolumn{3}{|c|}{$\begin{array}{l}\text { Exemple } \\
\text { GAL du Velay } \\
115\end{array}$} \\
\hline & $\begin{array}{c}\text { Points de } \\
\text { pondération }\end{array}$ & $\begin{array}{l}\text { Nombre de dos- } \\
\text { siers répondant } \\
\text { aux critères }\end{array}$ & Calcul & $\begin{array}{c}\text { Points de } \\
\text { pondération }\end{array}$ & $\begin{array}{l}\text { Nombre } \\
\text { de dossiers } \\
\text { répondant } \\
\text { aux critères }\end{array}$ & Calcul \\
\hline Enjeu agriculture & a (1 à 3) & $\mathrm{v}$ & $v * a=A$ & 2 & 10 & 20 \\
\hline Enjeu hors agriculture & b (1 à 3) & w & $w * b=B$ & 2 & 33 & 66 \\
\hline Enjeu services & C (1 à 3) & $x$ & $x * C=C$ & 1 & 8 & 8 \\
\hline Enjeu patrimoine naturel & 1 & $\mathrm{y}$ & $y * 1=D$ & 1 & 28 & 28 \\
\hline Enjeu patrimoine culturel & 1 & $\mathrm{z}$ & $z * 1=E$ & 1 & 43 & 43 \\
\hline \multicolumn{2}{|l|}{ Ancrage territorial (V1) } & $A+B+C+D+E=\sum$ & $\left.\sum / \mathrm{n}=\mathrm{DV}_{\mathrm{i}} \mathrm{i}^{*}\right)$ & & 166 & 1,4 \\
\hline \multicolumn{2}{|c|}{ Intersectorialité (V2) } & & $\mathrm{DV} 2_{\mathrm{i}}$ & & & 1,9 \\
\hline \multicolumn{2}{|c|}{ Coordination entre acteurs (V3) } & & DV3 $_{i}$ & & & 0,7 \\
\hline \multicolumn{2}{|c|}{ Transversalité (V4) } & & DV $4_{i}$ & & & 3,4 \\
\hline \multicolumn{2}{|c|}{ Degré de territorialisation de i } & \multicolumn{2}{|c|}{$=D V 1_{i}+D V 2_{i}+D V 3_{i}+D V 4_{i}$} & & & 7,4 \\
\hline
\end{tabular}

Note : $\left(^{*}\right) D V=$ Degré de la Variable

Source : les auteurs.

10. Le Pays du Velay regroupe 94000 habitants situés autour de la ville du Puy-en-Velay en Haute-Loire. 
la pondération est évaluée par projet. Elle est donc variable sur un même territoire.

L'intersectorialité de chaque projet est mesurée par trois variables : le nombre de cofinanceurs, le nombre de thématiques concernées, et le fait que l'opération soit innovante ou pas pour le territoire. La pondération des deux premières variables s'étale de 0 à 3 , par exemple 0 si 1 thématique est concernée, 1 pour 2 thématiques, 2 pour 3 thématiques, 3 pour plus de 3 thématiques concernées. La pondération de la troisième variable va de 0 à 2 .

La coordination entre acteurs est évaluée en fonction des réponses aux trois questions suivantes : le projet vise-t-il spécifiquement à coordonner les acteurs du territoire ? Le projet est-il intégré à d'autres projets portés par le même maître d'ouvrage ? Le projet entre-t-il dans une dynamique de territoire ou y a-t-il d'autres projets du même type portés sur le territoire par d'autres maîtres d'ouvrage ? Tout projet répondant à l'une des deux premières questions est pondéré par 3, et de 2 lorsqu'il répond positivement à la dernière question.

Pour la transversalité, les projets conduits au sein de chaque programme Leader répondant à 1 dimension du développement durable sont pondérés par 2 , ceux répondant à 2 dimensions du développement durable par 4 , et enfin ceux répondant à 3 dimensions du développement durable sont pondérés par 8.

Les pondérations ont été retenues de façon à obtenir un relatif équilibre entre les quatre dimensions principales du degré de territorialisation.

Puis, la méthode de calcul (tableau 2) a été appliquée afin d'agréger les contributions des quatre leviers à l'évaluation du degré de territorialisation.

Du fait de la méthode utilisée, cette analyse ne prend en compte que les dossiers bénéficiant d'un cofinancement Feader, et elle se limite aux données quantifiables disponibles, qui représentent de façon peu satisfaisante certains critères : par exemple, la mise en réseau d'acteurs, phénomène très important sur le terrain, ne peut être estimée que partiellement par cette analyse sans des investigations qualitatives menées auprès des porteurs de projet dans le cadre d'études de cas. Ainsi, la méthode proposée est très complémentaire d'approches qualitatives comme l'ont souligné les résultats obtenus, notamment en termes de comparaison entre territoires.

\section{Territorialisation plus marquée mais faible du programme Leader}

La méthode a été appliquée pour tous les $\mathrm{GAL}^{11}$ des régions Auvergne et Bourgogne (ce qui représente 1342 et 522 projets engagés respectivement dans chacune des deux régions au 15 mars 2011). Un des résultats les plus marquants est la mise en évidence d'un degré de territorialisation moyen des projets Leader (estimé avec la méthode générale présentée dans le tableau $2(8,93))$ mais légèrement et significativement ${ }^{12}$ supérieur à celui des projets hors Leader $(8,02)^{13}$. La démarche Leader apporte une valeur ajoutée en termes de territorialisation mais celle-ci reste faible. De plus, les projets sont globalement plus territorialisés en Bourgogne qu'en Auvergne tant pour les projets conduits dans le cadre de Leader ou en dehors de ce cadre. Ainsi, le degré de territorialisation des projets axe 3 hors Leader bourguignons

11. L'application réalisée a concerné les 21 territoires organisés en GAL des deux régions. Afin de permettre la comparaison avec les projets LEADER axe 4, les dossiers axe 3 (gérés régionalement) sont répartis par GAL en fonction de la localisation des bénéficiaires des mesures.

12. Estimation avec un test de Student, la probabilité étant inférieure à $5 \%$.

13. Dans notre méthode, le score à l'échelle du degré de territorialisation peut varier en théorie entre 0 (degré de territorialisation très faible) et 32 (degré de territorialisation très élevé) en raison du choix des pondérations présenté dans le tableau 2. 
(moyenne de l'ordre de 9,1) est-il supérieur à celui de leurs homologues auvergnats (moyenne de l'ordre de 7,9). Il atteste d'un effet régional qui peut être lié à des effets d'apprentissage dans les démarches de territorialisation de politique publique concordantes avec la plus grande ancienneté de ce type de démarche en Bourgogne (depuis les années 1990). Enfin, dans les deux régions, le degré de territorialisation des projets portés par les parcs classés Parc naturel régional (PNR) sont supérieurs à ceux des autres territoires organisés (à l'exception du PNR des Volcans d'Auvergne, plus vaste PNR de France, dont le territoire a été « éclaté » entre plusieurs GAL).

\section{Des différences marquées de territorialisation selon les mesures}

Les projets sont plus ou moins territorialisés selon la nature de la thématique sur laquelle ils portent. Les projets favorisant les stratégies locales de développement de la filière forêt/bois (mesure 341A) sont les plus territorialisés ; en effet, il s'agit notamment d'élaboration/animation de plans de massif ou chartes forestières, qui associent généralement une composante économique, une composante environnementale, voire une composante sociale. De tels projets font donc fortement appel au levier de la transversalité de par leur nature mais également à ceux relatifs au caractère intersectoriel et de coordination entre acteurs en raison de l'existence d'une composante animation/formation significative. De façon générale, ce dernier levier d'animation/formation est le moins sollicité, mais c'est aussi certainement le plus difficile à évaluer : il est activé par les actions par nature coordinatrices comme les 341B (stratégies locales de développement hors filière forêt/bois), ou 323A (élaboration et animation de Docob), et par les actions qui s'inscrivent dans un projet plus large du maître d'ouvrage ou du territoire.

Les projets favorisant la conservation et la mise en valeur du patrimoine naturel et culturel mesures (323D et E) ou bien la formation et l'information des acteurs socio-économiques (mesure 331), sont aussi fortement territorialisés mais leur champ d'action se limite aux leviers de l'intersectorialité et de la transversalité. Quant aux projets de diversification vers des activités non agricoles (mesure 311), d'aide à la création et au développement des microentreprises (mesure 312) et de services de base pour l'économie et la population
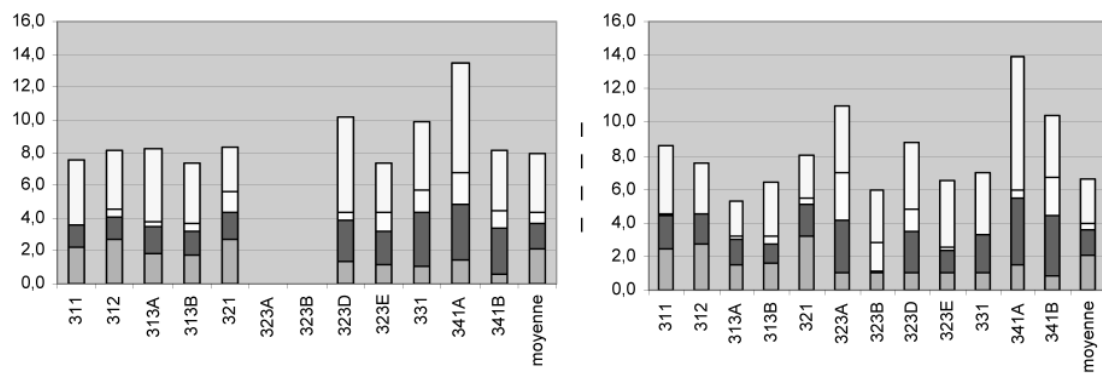

$\square$ Transversalité

$\square$ Coordination entre acteurs

口Intersectorialité

$\square$ Ancrage territorial 


\begin{tabular}{|c|c|}
\hline $\begin{array}{l}\text { La stratégie de développement du } \\
\text { GAL } \\
\text { Choix des mesures programmées : } \\
\text { sont plus ou moins territorialisées de par } \\
\text { leur nature. }\end{array}$ & $\begin{array}{l}\text { Mode de sélection } \\
\text { Dans certains cas, les critères de sélection } \\
\text { choisis ne peuvent permettre qu'aux } \\
\text { projets territorialisés d'émerger, ce qui } \\
\text { représente un frein pour les maîtres } \\
\text { d'ouvrage. Pour cette raison, un certain } \\
\text { nombre de projets ne sont pas déposés en } \\
\text { fait. Dans quel sens cela influe-t-il sur le } \\
\text { degré de territorialisation? }\end{array}$ \\
\hline \multicolumn{2}{|c|}{ Degré de territorialisation } \\
\hline $\begin{array}{l}\text { L'ancienneté du GAL ou de la structure } \\
\text { porteuse } \\
\text { Les GAL des premières générations de } \\
\text { programmes bénéficient de l'expérience et du } \\
\text { savoir-faire des acteurs, d'une ingénierie } \\
\text { aguerrie à Leader et de la construction des } \\
\text { projets sur un temps plus long favorable à une } \\
\text { plus forte territorialisation. }\end{array}$ & $\begin{array}{l}\text { Les conditions de structuration en GAL } \\
\text { II est courant d'opposer démarches ascendante } \\
\text { et descendante. Dans certains cas, les GAL se } \\
\text { constituent selon la volonté des acteurs locaux, } \\
\text { dans d'autres, les collectivités territoriales et les } \\
\text { services de l'État ont pu inciter les territoires à } \\
\text { se constituer en GAL. Cela influence les projets } \\
\text { car la motivation des acteurs est différente. On } \\
\text { peut discerner deux types de perception du } \\
\text { programme Leader : pour certains, il s'agit d'un } \\
\text { outil adapté à une politique de développement ; } \\
\text { d'autres n'intègrent pas la spécificité Leader et } \\
\text { l'utilisent alors comme un outil parmi d'autres. }\end{array}$ \\
\hline
\end{tabular}

rurale (mesure 321), ils actionnent le levier de la transversalité et de l'ancrage territorial de façon privilégiée.

\section{Une démarche de quantification interrogeant les processus de territorialisation à l'œuvre}

L'analyse du degré de territorialisation pour les projets hors Leader comme Leader met en évidence des différences marquées entre territoires organisés. La démarche quantitative proposée peut donc se révéler très utile pour illustrer ou renvoyer de nouvelles questions à une démarche d'analyse qualitative du processus de territorialisation. Même si la méthode proposée ici peut être dupliquée dans d'autres régions et peut suffire pour porter une première appréciation sur les projets Leader, elle gagne en pertinence à être croisée avec d'autres démarches plus qualitatives. Quelques exemples bourguignons et auvergnats rendent compte des processus de territorialisation à l'œuvre (figure 2).

En Bourgogne, les GAL de l'Auxois Morvan et du Centre Saône et Loire ont les plus forts degrés de territorialisation sur les projets Leader de Bourgogne. En Auxois Morvan, cette différence est-elle attribuable à la rigueur de la sélection des projets Leader ? En effet, afin de prétendre à Leader, les projets sont testés par une grille d'évaluation basée sur l'Agenda 21 du territoire. Ainsi, les projets éligibles doivent être à la fois transversaux, intersectoriels et ancrés au territoire. Le GAL du Centre Saône et Loire s'est constitué sous l'effet d'une 
réelle motivation et d'une forte demande de la part des acteurs locaux. Est-ce pour cette raison qu'il actionne tous les leviers de la territorialisation?

Les GAL auvergnats du Livradois Forez et des Combrailles ont également des degrés de territorialisation élevés. Ce niveau élevé est-il en lien avec l'expérience de ces territoires en matière d'ingénierie territoriale ? En termes d'intersectorialité, ce sont parmi les plus élevés, car ils ont une forte technicité dans le montage de leurs projets. La coopération entre les différents animateurs-coordonnateurs des GAL permet l'émergence de projets multithématiques et pluripartenariaux. Ces éléments constituent-ils les facteurs majeurs expliquant le degré élevé de territorialisation?

$*$

$* *$

Les politiques intégrées de développement rural de type Leader présentent une valeur ajoutée certaine par rapport aux politiques plus descendantes. Toutefois, bien que significative d'un point de vue statistique, la différence est faible et très variable selon les mesures et les territoires. Cette variabilité interterritoriale trouve son origine essentiellement dans les effets d'apprentissage (eux-mêmes liés notamment à l'ancienneté des pratiques). Toutefois, il semble bien que l'intégration de Leader dans le Plan de développement rural hexagonal (PDRH) ait véritablement freiné les capacités d'innovation territoriale (contrairement à la génération précédente de Leader) notamment pour les porteurs de projets privés confrontés aux difficultés de trouver un cofinancement. Elle semble cependant avoir joué un rôle de diffusion au sein des territoires organisés aiguillant les projets selon leurs caractéristiques à divers guichets, tout en essayant d'accroître l'intégration territoriale de tous les projets grâce aux exemples et bonnes pratiques mises en avant dans les démarches Leader. Il en résulte une valeur ajoutée territoriale de Leader du fait d'un effet de diffusion vers l'ensemble des territoires ruraux, comme nous avons effectivement pu l'observer en Bourgogne.

De plus, en s'appuyant sur un cadre théorique de la territorialisation des politiques publiques, la démarche quantitative proposée ici présente de nombreux intérêts tant pour les scientifiques que pour les décideurs publics. Pour les décideurs publics, elle représente une première modalité d'appréciation de la valeur ajoutée des politiques territoriales de type Leader, comme l'a montré la première application réalisée en Auvergne et Bourgogne. Elle peut aussi être utilisée dans le cadre plus général de l'évaluation de telles politiques, que ce soit in itinere ou ex post mais également pour d'autres politiques territorialisées disposant d'une base de données de ce type (par exemple, des contrats de développement). La démarche proposée s'avère très complémentaire de celles mises en œuvre par les économistes et les politistes pour rendre compte de la diversité des processus de territorialisation à l'œuvre. Toutefois, la généralisation hors des régions étudiées ici nécessiterait probablement des observations qualitatives complémentaires.

Lors de la mise en place des programmations 2007-2013, et notamment des projets Leader, des stratégies différentes ont pu être observées selon les régions, entre une forte consommation de crédits dès le démarrage comme en Auvergne, ou bien une mise en route plus lente comme en Bourgogne. Ceci pose différentes questions : les projets dont le mode de sélection est plus contraignant sont-ils nécessairement plus territorialisés ? Les projets plus territorialisés sont-ils plus pertinents pour le territoire ? Il s'agit là d'autant de questions interrogeant à la fois le scientifique et le praticien.

Cet article est tiré des travaux menés dans le cadre du programme de recherche Pour et Sur le Développement Régional - Projet Regiab financé par I'INRA, I'Irstea, le CIRAD et les régions Auvergne et Bourgogne. 